Proceedings of the CSMAG'07 Conference, Košice, July 9-12, 2007

\title{
Spin Glass-Like Transition in Orthorhombic T-Phase Al-Pd(Fe)-Mn Complex Metallic Alloys
}

\author{
J. SlanoveC
}

Iskraemeco, d.d., Savska loka 4, 4000 Kranj, Slovenia

Z. Jaghičić, M. Jagodič, Z. Trontelu

Institute of Mathematics, Physics and Mechanics

Jadranska 19, 1000 Ljubljana, Slovenia

\section{Heggen, M. Feuerbacher, S. Balanetskyy}

Institut für Festköperforschung, Forschungszentrum Jülich GmbH 52425 Jülich, Germany

AND J. DOLINŠEK

FMF and Jožef Stefan Institute, Jamova 39, 1000 Ljubljana, Slovenia

Magnetic properties of orthorhombic T-phase Al-Pd(Fe)-Mn - members of complex metallic alloys - with different $\mathrm{Pd}(\mathrm{Fe}) / \mathrm{Mn}$ atomic ratios were studied experimentally. The measured zero field cooling-field cooling splitting, the frequency dependent AC susceptibility and slow thermoremanent magnetization time decay indicate that the samples possess built-in frustration-like properties similar to those in spin glasses.

PACS numbers: $75.10 . \mathrm{Nr}, 74.25 . \mathrm{Ha}, 71.23 . \mathrm{Ft}$

\section{Introduction}

Complex metallic alloys (CMAs) denote intermetallic phases whose giant unit cells contain from many tens up to more than thousand atoms. Inside the giant unit cell the atoms are arranged in clusters with local icosahedral symmetry. Consequently, in CMAs there exist two substantially different physical length scales - so that interesting physical properties may appear (e.g. temperature independent electrical conductivity).

\section{Experimental}

We have studied magnetic properties of six polygrain samples - members of CMAs — of orthorhombic T-phase $\mathrm{Al}-\mathrm{Pd}(\mathrm{Fe})-\mathrm{Mn}$ with local pentagonal symmetry [1] grown by the Bridgman technique. All samples have the 
same structure but different compositions: a binary $\mathrm{Al}_{73} \mathrm{Mn}_{27}$ and the ternaries $\mathrm{Al}_{73} \mathrm{Mn}_{27-n} \mathrm{Pd}_{n}$ and $\mathrm{Al}_{73} \mathrm{Mn}_{27-n} \mathrm{Fe}_{n}(n=2,4,6$, shortly AMP- $n$ and AMF- $n$ ). The chemical compositions were determined by powder X-ray diffraction.

Magnetic properties have been investigated with a Quantum Design SQUID magnetometer MPMS-XL-5. Susceptibility between room temperature and $100 \mathrm{~K}$ follows the Curie-Weiss law $\chi=C /(T-\theta)$. From the Curie constants (Table) we calculated the average magnetic moments per magnetic ion. The average magnetic

\section{TABLE}

Curie constant $C$, average magnetic moment per magnetic atom and Curie-Weiss temperature.

\begin{tabular}{c|c|c|c}
\hline \hline & $\begin{array}{c}C \\
{[\mathrm{emu} \mathrm{K} / \mathrm{mol}]}\end{array}$ & $\begin{array}{c}\bar{\mu}_{\mathrm{eff}} \\
{\left[\mu_{\mathrm{B}}\right]}\end{array}$ & $\begin{array}{c}\theta \\
{[\mathrm{K}]}\end{array}$ \\
\hline $\mathrm{Al}_{73} \mathrm{Mn}_{27}$ & 25.9 & 2.8 & -11 \\
$\mathrm{AMF}-2$ & 22.2 & 2.6 & -33 \\
$\mathrm{AMF}-4$ & 22.6 & 2.6 & -40 \\
$\mathrm{AMP}-2$ & 22.0 & 2.7 & -32 \\
$\mathrm{AMP}-4$ & 20.2 & 2.6 & -10 \\
$\mathrm{AMP}-6$ & 18.1 & 2.6 & -23
\end{tabular}

moments $2.7 \mu_{\mathrm{B}}$ for all six samples are reduced by a factor of 2 with respect to the values expected for the bore $\mathrm{Mn}$ and Fe ions. This indicates that magnetic moments are partially screened by conduction-electron cloud in a conducting environment. The results of a theoretical study of the distribution of magnetic moments over the lattice [2] show that three of the Mn sites carry substantial moments, whereas all other Mn sites carry only small magnetic moments. Consequently the magnetic structure lies somewhere between a random structure as in canonical spin glasses and an ordered but nonperiodic structure as in rare-earth containing quasicrystals. On the other hand, a relatively large negative Curie-Weiss temperature $\theta$ (ranging between $-10 \mathrm{~K}$ and $-40 \mathrm{~K}$ for different samples) indicates an antiferromagnetic (AFM) interaction between magnetic moments. AFM interaction together with a nonperiodic structure may lead to a frustration-like magnetic properties at low temperatures. The aim of our research is to describe and understand magnetic properties of the T-phase $\mathrm{Al}-\mathrm{Pd}(\mathrm{Fe})-\mathrm{Mn} \mathrm{CMAs}$ in the light of their unique magnetic structure.

\section{Results and discussion}

The low temperature susceptibility (Fig. 1) measured in a magnetic field of $10 \mathrm{mT}$ shows a large zero field cooling-field cooling (ZFC-FC) splitting indicating a phase transition at freezing temperature $T_{\mathrm{f}}$. The freezing temperature determined from the maximum in ZFC susceptibility is about $22 \mathrm{~K}$ for the binary 
phase $\mathrm{Al}_{73} \mathrm{Mn}_{27}$ and ternaries with Fe atoms while for the Pd containing samples $T_{\mathrm{f}}$ changes with the $\mathrm{Pd}$ contents. The FC susceptibility below $T_{\mathrm{f}}$ is almost temperature independent as is usually observed in spin glasses.
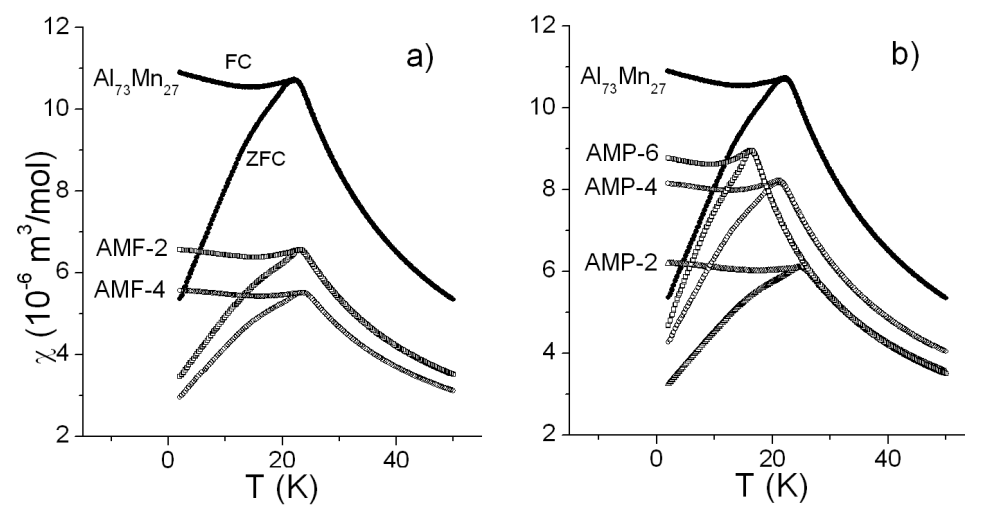

Fig. 1. ZFC and FC susceptibilities measured in magnetic field of $10 \mathrm{mT}$. For comparison the data for the binary sample $\mathrm{Al}_{73} \mathrm{Mn}_{27}$ are plotted in both (a) and (b) figures.

AC susceptibility was measured at four frequencies of applied field: $1 \mathrm{~Hz}$, $10 \mathrm{~Hz}, 100 \mathrm{~Hz}$, and $1 \mathrm{kHz}$. The results for the $\mathrm{Al}_{73} \mathrm{Mn}_{27}$ sample are shown in Fig. 2. The temperature of the maximum increases with frequency of the applied magnetic field. In the inset to Fig. 2 a relative shift of $\Delta T_{\mathrm{f}} / T_{\mathrm{f}}$ as a function of $\log v$ is plotted. The dependence is linear with $\Delta T_{\mathrm{f}} /\left(T_{\mathrm{f}} \Delta \log v\right)=0.01$. This value falls in the range expected for canonical spin glasses and is about 10 times smaller than in typical superparamagnets [3]. Similar dependences were observed for all other samples.

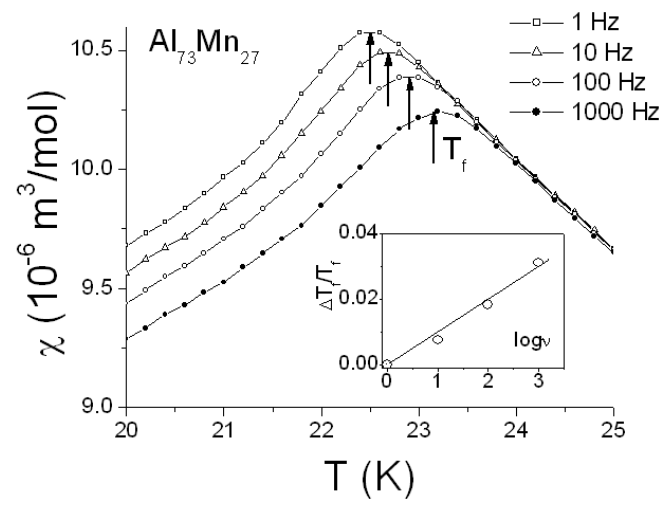

Fig. 2. AC susceptibility and shift of the $T_{\mathrm{f}}$ with frequency for the $\mathrm{Al}_{73} \mathrm{Mn}_{27}$ sample.

Finally, we have searched for the slow thermoremanent magnetization (TRM) time decay that is a typical characteristic of all frustrated systems. An 
experiment is shown in Fig. 3. The sample is cooled in a low magnetic field $H$ from a temperature well above $T_{\mathrm{f}}$ to a measuring temperature $T_{\mathrm{m}}$ where the cooling is stopped for a waiting time $t_{\mathrm{w}}$ : this way the spin system is let to approach an equilibrium state in the field $H$. After the waiting time $t_{\mathrm{w}}$ the field is turned down to zero, which results in an almost instantaneous decay of the reversible part of the magnetization, followed by a slow decay of the irreversible part (TRM) of the magnetization. The slow TRM decay is a consequence of high barriers in the free-energy landscape of a frustrated system and has been observed in all studied samples. The amplitude of TRM as a function of waiting time and measured temperature was similar as in other frustrated systems (for example spin glasses [3] or some quasicrystals [4]).

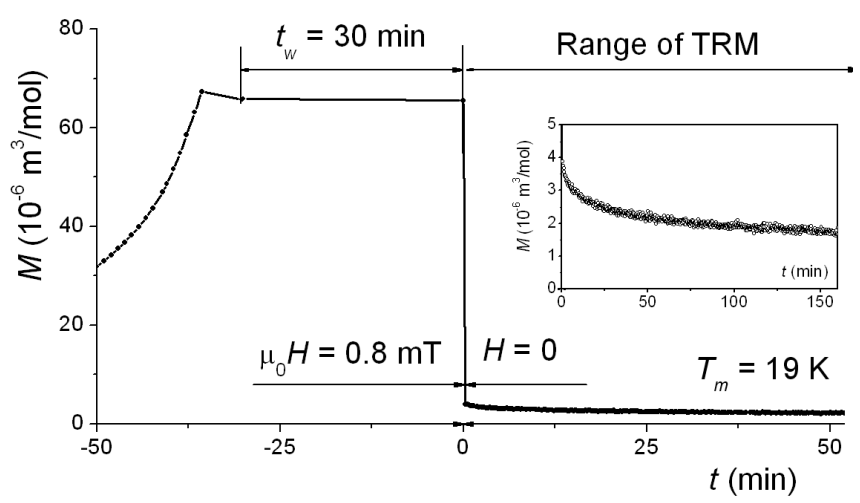

Fig. 3. A typical TRM experiment measured in the sample AMP-4. Slow TRM decay is shown in the inset with expanded $y$-scale.

\section{Conclusion}

In conclusion, the measured ZFC-FC splitting, the frequency dependent $\mathrm{AC}$ susceptibility and slow TRM decay indicate that the orthorhombic T-phase Al$\mathrm{Pd}(\mathrm{Fe})-\mathrm{Mn}$ quasicrystalline approximants possess built in frustration-like properties similar to those in spin glasses.

\section{References}

[1] H. Klein, M. Boudard, M. Audier, M. de Boissieu, H. Vincent, L. Beraha, M. Duneau, Philos. Mag. Lett. 75, 197 (1997).

[2] J. Hafner, M. Krajči, Phys. Rev. B 75, 2849 (1998).

[3] J.A. Mydosh, Spin Glasses: an Experimental Introduction, Taylor \& Francis, London 1993, p. 67.

[4] J. Dolinšek, Z. Jagličić, M.A. Chernikov, I.R. Fisher, P. Canfield, Phys. Rev. B 64, 224209 (2001). 